\title{
Tangential derivative mapping of axial MEG applied to event-related desynchronization research
}

\author{
Marcel C.M. Bastiaansen ${ }^{\text {a, b,*}}$, Thomas R. Knösche \\ ${ }^{\mathrm{a}}$ Cooperation Centre Tilburg and Eindhoven Universities, Tilburg, The Netherlands \\ ${ }^{\mathrm{b}}$ Psychonomics section, Tilburg University, P.O. Box 90153, 5000 LE Tilburg, The Netherlands \\ ${ }^{\mathrm{c}}$ Max-Planck-Institute of Cognitive Neuroscience, Leipzig, Germany
}

Accepted 2 February 2000

\begin{abstract}
Objectives: A problem with the topographic mapping of MEG data recorded with axial gradiometers is that field extrema are measured at sensors located at either side of a neuronal generator instead of at sensors directly above the source. This is problematic for the computation of event-related desynchronization (ERD) on MEG data, since ERD relies on a correspondence between the signal maximum and the location of the neuronal generator.

Methods: We present a new method based on computing spatial derivatives of the MEG data. The limitations of this method were investigated by means of forward simulations, and the method was applied to a 150-channel MEG dataset.

Results: The simulations showed that the method has some limitations. (1) Fewer channels reduce accuracy and amplitude. (2) It is less suitable for deep or very extended sources. (3) Multiple sources can only be distinguished if they are not too close to each other. Applying the method in the calculation of ERD on experimental data led to a considerable improvement of the ERD maps.

Conclusions: The proposed method offers a significant advantage over raw MEG signals, both for the topographic mapping of MEG and for the analysis of rhythmic MEG activity by means of ERD. (C) 2000 Elsevier Science Ireland Ltd. All rights reserved.
\end{abstract}

Keywords: Event-related desynchronization; Simulations; MEG; Axial gradiometers; Spatial derivatives; Voluntary movement

\section{Introduction}

Event-related desynchronization (ERD; Pfurtscheller and Aranibar, 1977) is a technique used to quantify the spatiotemporal evolution of event-related changes in oscillatory EEG activity. ERD has proved to be a sensitive indicator of cortical activity in movement-related brain research as well as in cognitive brain research (cf. Pfurtscheller and Lopez da Silva, 1999, for a comprehensive review of the state of the art of ERD research).

Because of the inherently better spatial resolution of MEG signals as compared to EEG, and because of the differential sensitivity of EEG and MEG for radial and tangential dipoles, it would be desirable to apply the ERD technique to MEG measurements as well. There is, however, a problem with the straightforward computation of ERD on certain types of MEG data. Many modern MEG systems use pickup coils that are sensitive to the component of the magnetic field which is approximately normal to the

\footnotetext{
* Corresponding author.

E-mail address: marcel.bastiaansen@mpi.nl (M.C.M. Bastiaansen).
}

head surface. As a consequence, a dipole at a given location will produce maximum signal at either side of the dipole, while just above the source the signal will be zero. ERD mapping relies on a reasonable correspondence between the maximum signal and the location of the activated brain area. Since the extrema of the normal components of the magnetic field can be quite distant from the location of the activation, the interpretation can be difficult.

With MEG systems that use so-called planar gradiometers (where the pick-up coil and the compensation coil are in the same plane, which is tangential to the scalp surface) this problem does not arise. Here, a dipole at a given location produces maximal signal at the sensors overlying it. Therefore MEG measured with planar gradiometers is well suited for topographic analyses such as ERD. It has been shown that ERD (or, more precisely, Temporal-Spectral Evolution (TSE; Salmelin and Hari, 1994), a method that uses a slightly different way of quantifying changes in oscillatory activity) can be successfully applied to 'planar' MEG data (see Hari and Salmelin, 1997, for a review). This has, amongst others, led to new insights about the nature and the generators of the mu and beta rhythms (e.g. Salmelin et 
al., 1995a,b), and has revealed the existence of $10 \mathrm{~Hz}$ rhythmic activity originating from the auditory cortex (Tiihonen et al., 1995; Lehtelä et al., 1997). These findings demonstrate that ERD analyses on MEG data do have an added value compared to ERD analyses on EEG data.

In the present paper we propose a solution to the problem of computing ERD on MEG data recorded with an axial gradiometer system. We have developed a method that is based on computing spatial derivatives of the recorded MEG. ERD can then be computed on the spatial derivatives instead of on the untreated MEG. We will present results from forward simulations that will give us an indication of the limitations and possibilities of the proposed method, and we will apply it in the calculation of ERD on a 150-channel MEG dataset in order to evaluate its usefulness in ERD research.

\section{Methods}

\subsection{Computation of $M E G$ derivatives}

The normal component of the magnetic field, which is measured by axial gradiometers or magnetometers is considered a scalar field on a surface defined by the sensor array: $B(u, v)$. The local coordinates $\mathrm{u}$ and $\mathrm{v}$ are locally tangential and mutually orthogonal. The gradient of $B$ denotes the direction and the steepness of the slope of $B$ within the sensor surface:

$\nabla B(u, v)=(\partial B / \partial u, \partial B / \partial v)^{T}$

The steepness of the slope of $B(u, v)$, i.e. the density of contour lines in a contour plot, is the measure we want to express. The reason for this is that the field gradient is largest just above the generator (cf. Fig. 1a). Therefore, the new measure is defined as the Euclidian norm of the abovementioned gradient:

$\|\nabla B(u, v)\|=\operatorname{sqrt}\left((\partial B / \partial u)^{2}+(\partial B / \partial v)^{2}\right)$

The derivatives are computed using a 3D spline interpolation of $B(u, v)$.

Spatial derivatives, no matter whether computed or directly measured using planar gradiometer arrangements, work as spatial high-pass filters. This has several consequences. First of all, the signal-to-noise ratio (SNR) is decreased. Hence, if a spatial derivative is applied, we have to make sure that the SNR is not too poor initially. Furthermore, suitable regularization should be applied to the derivation procedure. A second consequence of using a derivative is that sharp, distinct features in the signal are favored over smoother structures. That means, the method is expected to be particularly sensitive to focal and superficial sources.

\subsection{Simulations}

For the simulations we used a spherical head model. Test source configurations were used to simulate MEG measurements. Then the spatial derivatives of the MEG were computed with the software package ASA (A.N.T. Software B.V., the Netherlands).

Seven simulation parameters were systematically varied: (1) relative source depth as distance between sensors and source divided by the distance between sensors and the middle of the head $(0.36,0.52,0.68)$; (2) number of sources (1 and 4); (3) distance between two sources (4 and $10 \mathrm{~cm}$ ); (4) the mutual orientation of multiple sources (see Fig. 1c); (5) focality of the sources (focal sources and current sheets of extent 1, 2, 3, and $6 \mathrm{~cm}^{2}$ ); (6) signal-to-noise ratio (SNR) ${ }^{1}$ defined as signal maximum divided by the standard deviation of the noise $(20,10,5,2,1)$; and (7) number of sensors (64 and 150). Simulations with noise were repeated 20 times with different noise realizations. A result was considered stable if the maxima of the derivative map could be correctly identified for all noise realizations.

\subsection{MEG recordings and experimental set-up}

For the MEG recordings a 150-channel whole-cortex MEG system (CTF Systems Inc., Vancouver, Canada) was used. The axial gradiometers $(2 \mathrm{~cm}$ coil diameter and $5 \mathrm{~cm}$ baseline) are uniformly distributed on the helmet surface with mean spacing of $3.1 \mathrm{~cm}$.

Six healthy, paid volunteers participated in the experiment. They were seated in a dimly illuminated, soundattenuating and magnetically shielded chamber, and were asked to perform simple self-paced flexions of the index finger, with a rate of approximately 4-6 movements per minute. For each subject, approximately 100 MEG epochs from $3000 \mathrm{~ms}$ pre-movement to $3000 \mathrm{~ms}$ post-movement were digitized at $250 \mathrm{~Hz}$, and stored for off-line analysis. Filter settings were from DC to $45 \mathrm{~Hz}$, with additional notch filters at 50 and $60 \mathrm{~Hz}$. For each subject, we determined the position of the sensors relative to the subject's head on the basis of three head localization coils attached to the nasion and both pre-auricular points. Subsequently, we computed the average of the sensor positions for all subjects, and interpolated all the MEG data to these average sensor positions using the algorithm developed by Hämäläinen and Ilmoniemi (1994). The data were visually inspected for eye-movement and other artifacts, and contaminated epochs were discarded from further analysis. From the remaining epochs two datasets were created: one time-locked to movement onset for the computation of the pre-movement ERD, and the other time-locked to movement offset for the computation of the post-movement ERS.

\subsection{ERD computation}

The movement onset data were filtered in the $8-12 \mathrm{~Hz}$

\footnotetext{
${ }^{1}$ Along the rules of elementary mathematics, the average of a set of derivatives is equal to the derivative of the average. Therefore in the simulations we used SNR values that are typical for MEG data averaged over epochs: although for ERD computation the derivatives are computed at the single epoch level, the ERD is subsequently averaged over epochs.
} 
a) Influence of spatial sampling

\section{simulation results}
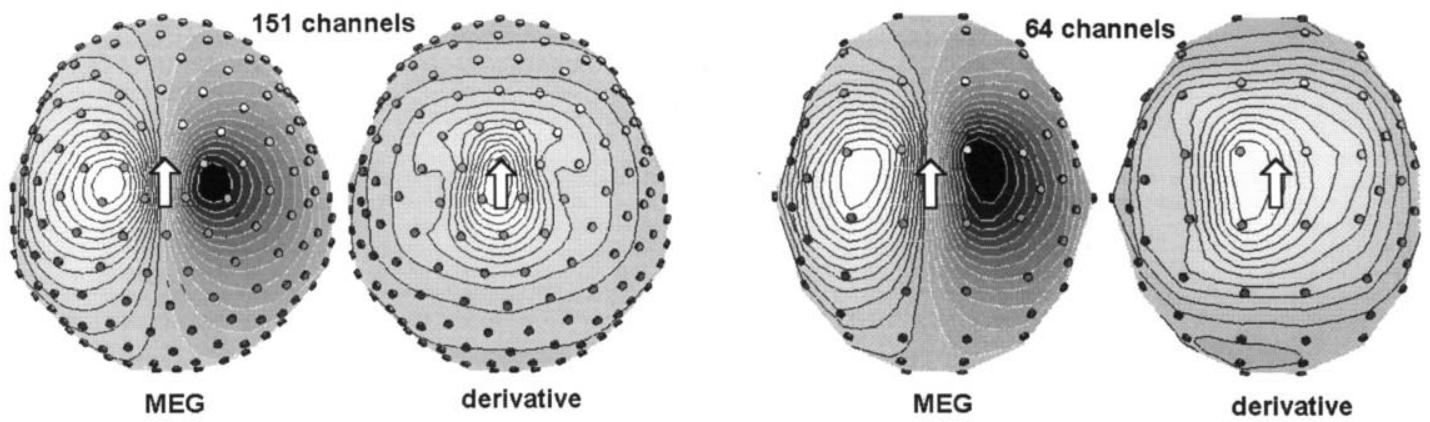

b) Influence of noise as a function of source depth and spatial extent
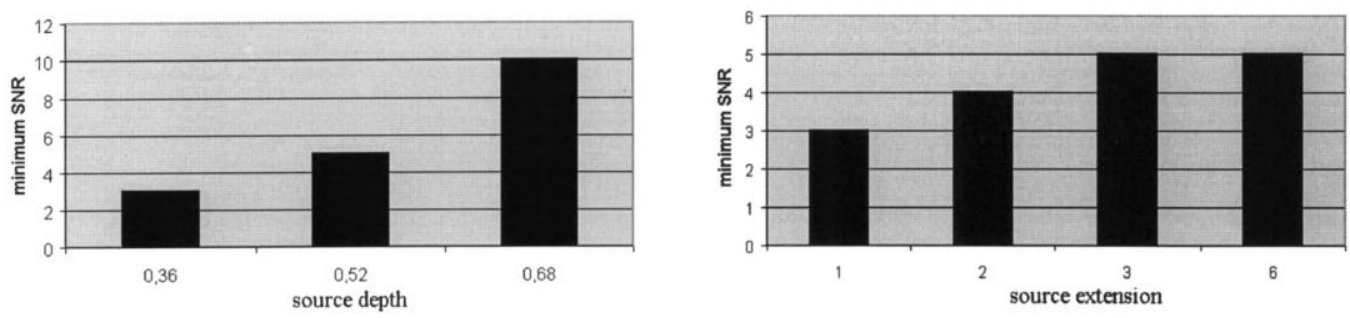

c) detectability of multiple focal sources
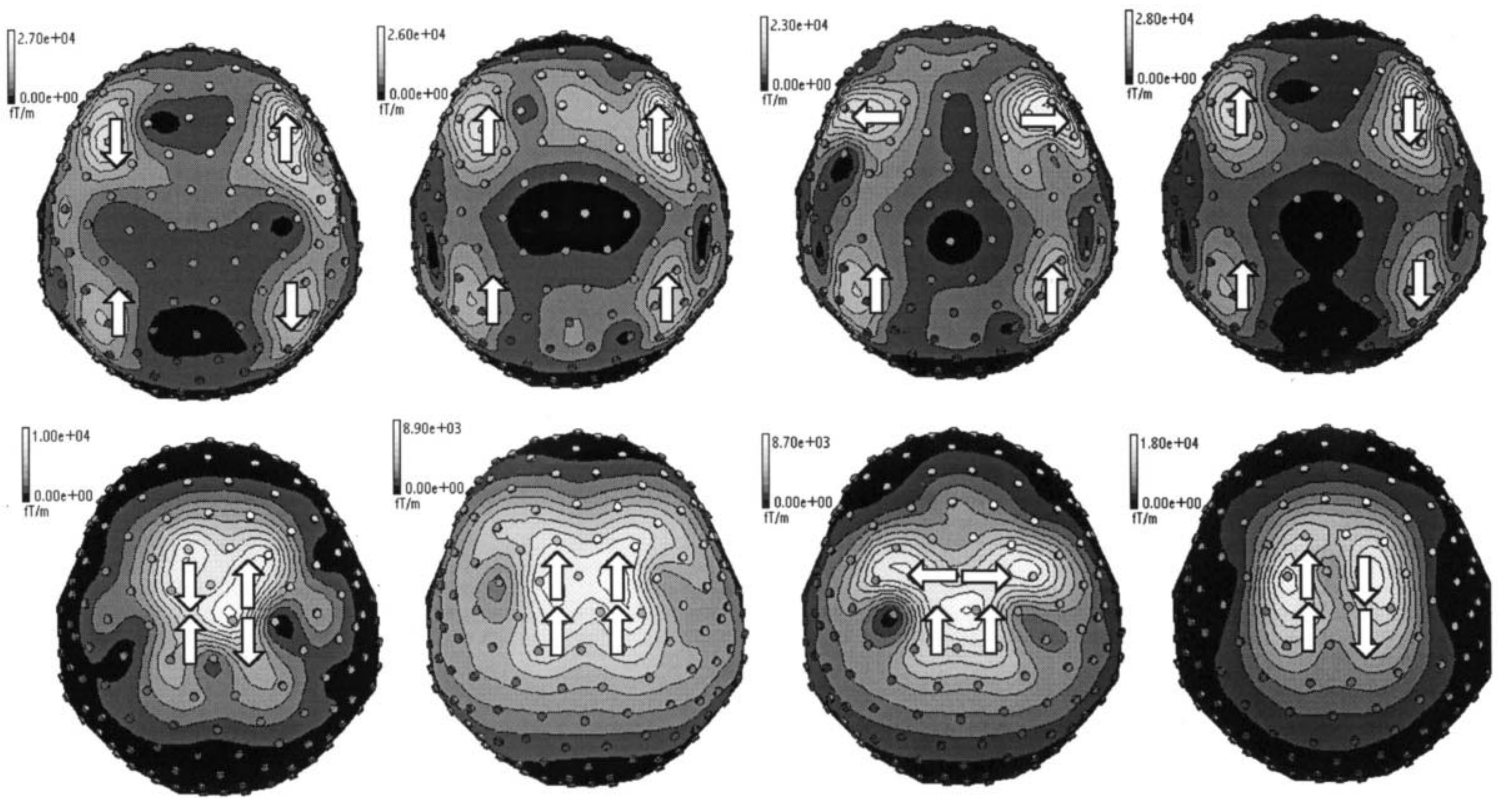

Fig. 1. Results of the forward simulations. (a) Maps of untreated MEG and of spatial derivatives for 64 and 150 sensors. White arrows indicate the position of the generating source. (b) Critical values of signal-to-noise ratio (SNR) at which sources can still be reliably identified, presented separately for different source depths (left) and different source extensions (right). (c) Maps of MEG derivatives showing the detectability of multiple focal sources for different source configurations. White arrows represent source locations and orientations.

band, and the movement offset data were filtered in the 17$23 \mathrm{~Hz}$ band. Next, MEG derivatives were computed using the method described above. This step was performed after the filtering, because using the absolute values of the derivatives (which is necessary in order to avoid cancellation of gradients with opposite signs) changes the frequency content of the MEG signal. The subsequent steps were performed both on the untreated, filtered MEG and on the derivatives of the filtered MEG. The data were squared, and averaged over intervals of $250 \mathrm{~ms}$. For each subject data 
were averaged over trials, and ERD was computed as the percentage power increase (event-related synchronization, ERS) or decrease (event-related desynchronization, ERD) for a particular time interval in a particular frequency band, relative to a pre-movement reference interval.

\section{Results}

\subsection{Simulations}

A first set of simulations, investigating the influence of the spatial sampling (number of sensors), noise, and source depth on the detectablility of focal sources revealed that both tested sensor configurations were capable of detecting a single dipole. However, lower sampling resolutions decrease the accuracy of the exact peak location. Due to the undersampling with only 64 sensors, the rather sharp peak above the source location cannot be properly reconstructed by the spline interpolation, reducing its amplitude. Since we define the SNR as the ratio of the peak of the signal amplitude and the standard deviation of the noise, this effectively diminishes the SNR, which explains the poorer correspondence between the location of the source and the maximum of the MEG derivative (see Fig. 1a). Moreover, we noticed that for very deep dipoles, side maxima occur. This is readily explainable, since the source is now evenly near to more than one region of the sensor array. Due to the low amplitude of signals from the depth (for 150 channels, the signal amplitude produced by a source at a relative depth of 0.68 is less than $10 \%$ of that produced by a source at a relative depth of 0.36 ), these artifacts will be masked by noise and more prominent superficial activity in most practical cases. Additionally, the SNR necessary for the detection of a single source increases with depth (see Fig. 1b), adding to the relative invisibility of deeper sources. These effects also underline the necessity of a small distance between head surface and sensors, especially near the brain regions of interest. Note that for the performance of the derivative method, the relative depth, i.e. the distance between sensors and source divided by the distance between the sensors and the middle of the head, is relevant. This value is influenced not only by the anatomical location of the source, but also by the sizes of the head and the sensor array. In a small head, all sources appear deeper.

In a second set of simulations, the influence of noise on the localizability of extended sources was explored. The tangential extent of a (superficial) source had a smaller effect than expected. The SNR necessary to localize the center of mass of the source reliably was similar to the value found with a focal source, except for the $6 \mathrm{~cm}^{2}$ generator. In this case, e.g. if the cortex of an entire lobe is active, an SNR of 8 was necessary (see Fig. 2b).

The third set of simulations dealt with the detection of multiple focal sources. In Fig. 2c, the used source configurations are depicted. They involve different mutual orientations and distances. The simulations revealed that mutual distances of a few centimeters (here $4 \mathrm{~cm}$ ) do not allow for a reliable resolution. However, multiple sources in different brain regions can be distinguished.

\subsection{Experimental data}

As a first step, in order to determine whether the quality of the experimental data is sufficient for the use of the proposed method, we determined the SNR separately for the movementonset data and for the movement-offset data. For both datasets, the SNR was 4.5, which according to the simulation results (cf. Fig. 1b) should be sufficient for a successful application of the derivatives method. In Fig. 2 the ERD and ERS on untreated MEG and on MEG derivatives is presented. When comparing both methods, three things should be noted. First, the location of the maximal ERD on untreated MEG data shows quite some variability over time, whereas the ERD on MEG derivatives has a high degree of spatial stability. This was verified by computing a measure of topological change between successive ERD time intervals, defined as 1 - absolute (correlation (map1, map 2)). A comparison of this measure between the ERD maps computed on the untreated MEG and on MEG derivatives, both for the pre-movement ERD and for the post-movement ERS (see Fig. 3a) confirmed the higher spatial stability of the ERD on MEG derivatives. Second, visual inspection of Fig. 2 indicates that both the pre-movement ERD and the post-movement ERS on MEG derivatives are more focal than the ERD/ERS on the untreated MEG. Moreover, the early (1000 to $500 \mathrm{~ms}$ pre-movement) ERD on the untreated MEG shows two maxima, roughly located at either side of the hand motor area, while the ERD on MEG derivatives shows only one focus which lies in between these maxima, and which nicely corresponds to the motor hand area. This is even more clearly seen in the post-movement ERS. Note that this phenomenon occurs also at the level of single-subject averages. In order to quantify the gain in focality resulting from the use of the MEG derivative, we computed the Global Field Power (GFP; Lehmann, 1971; see also Skrandies, 1995) for each ERD time interval, both for the pre-movement ERD and the post-movement ERS. As Fig. 3b illustrates, the GFP was indeed larger for the ERD on MEG derivatives for all ERD time intervals but one. Finally, just prior to as well as during the movement, the ERD on MEG derivatives reveals the bilateral ERD over both motor cortices that was expected on the basis of the results usually described in the literature (see e.g. Pfurtscheller and Berghold, 1989; Salmelin et al., 1995a,b; for a description of this effect on EEG and MEG data, respectively). In contrast, with untreated MEG, the two peaks are mislocated at much more medial sites over the frontocentral midline.

\section{Discussion}

The simulations showed that the method has some limitations, the most important being the requirement that simultaneously active sources should be at more than $4 \mathrm{~cm}$ apart in 


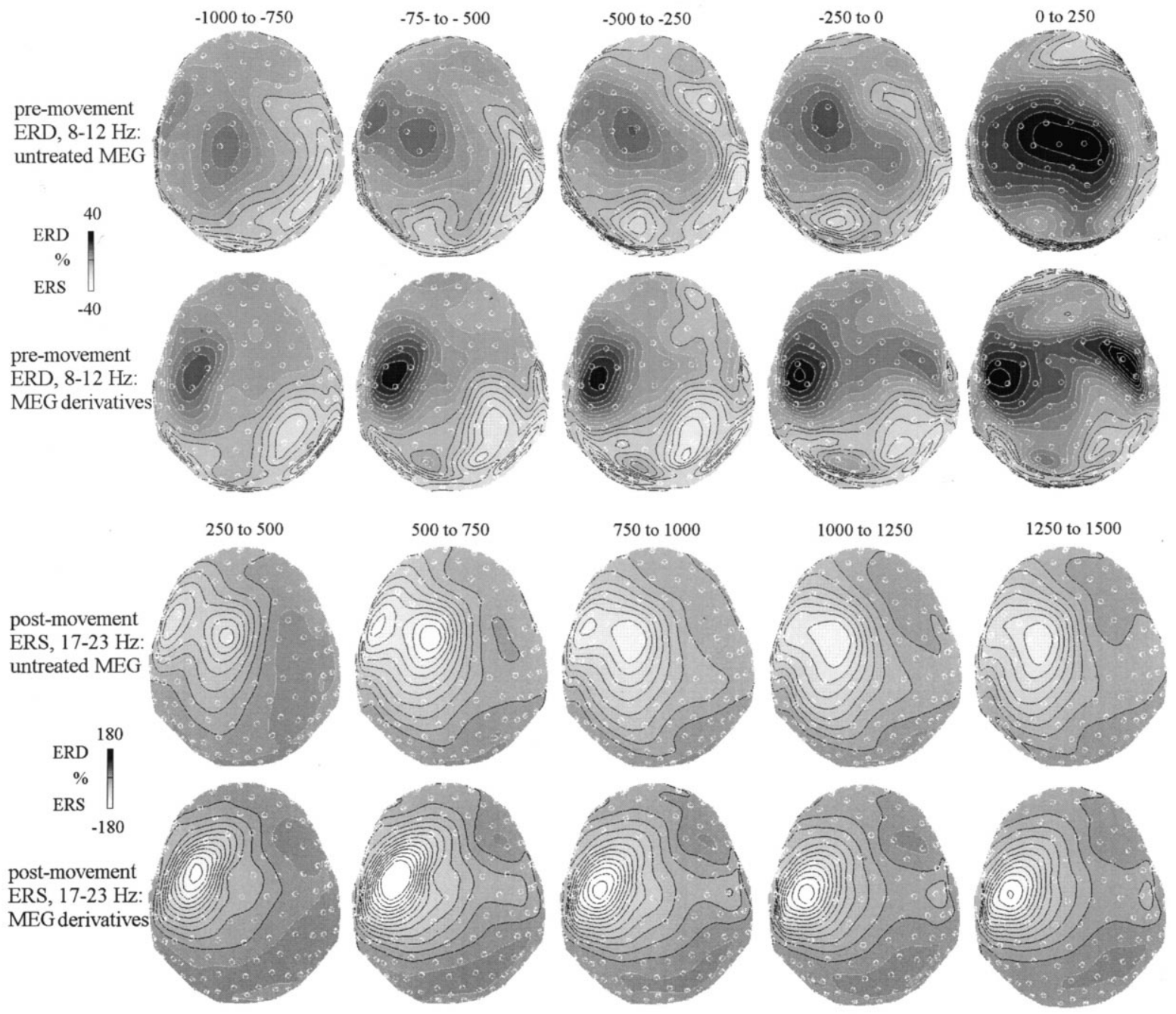

Fig. 2. Grand averages $(N=6)$ of pre-movement ERD in the 8-12 Hz frequency band (top rows) and post-movement ERS in the 17-23 Hz frequency band (bottom rows), on untreated MEG and on MEG derivatives. ERD, or power decrease, is depicted in dark gray surrounded by white contour lines, while ERS, or power increase, is depicted in light gray, surrounded by black contour lines. The gray contour lines correspond to zero power change.

order to be detected. Furthermore, the method is less suited for deep sources. Finally a dense sensor array increases the localization accuracy and the SNR. Applying the method as a first step in the calculation of ERD on experimental MEG data led to a higher spatial stability, and a more focal ERD and ERS that corresponded better to the hand motor areas for the MEG derivatives than for the untreated MEG.

These results indicate that it is possible to produce virtual planar MEG gradiometers from axial MEG data, which is particularly valuable for studying ERD/ERS effects. This method may also be useful for mapping axial MEG data in general. ERD of axial MEG signals has recently been the focus of interest of other research groups as well, and other methods for the calculation of ERD on MEG data are currently being developed (Babiloni et al., 1999; Edlinger et al., 1999). These methods aim at an integration of EEG and MEG by means of inverse linear estimation (LE) procedures (Edlinger et al., 1999), that can further be extended with fMRI-based constraints (Babiloni et al., 1999). The main advantage of using LE on a combination of EEG and MEG over the method proposed in the present paper is that LE makes optimal use of the complementary information that is present in EEG and MEG. Future research should point out whether this results in a significant enhancement of the spatial resolution of ERD. A practical problem of these methods is that they require the availability of simultaneously recorded EEG and MEG, and of structural MRI scans for the generation of realistic volume conduction models, while the method proposed by Babiloni et al. (1999) additionally requires availability of fMRI facilities. If such facilities are not available, the presently proposed method may be a valuable tool for investigating ERD on MEG data recorded with magnetometers or axial gradiometers.

\section{Acknowledgements}

The MEG experiment described in this paper was performed in close collaboration with the Department of 
a)

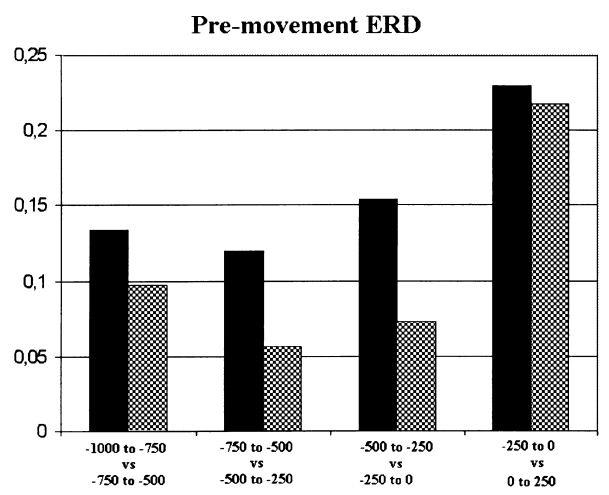

\section{Dissimilarity}

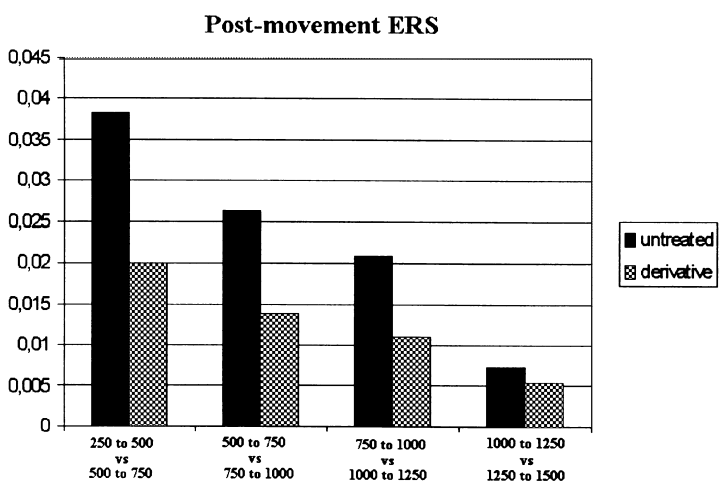

Global Field Power

b)

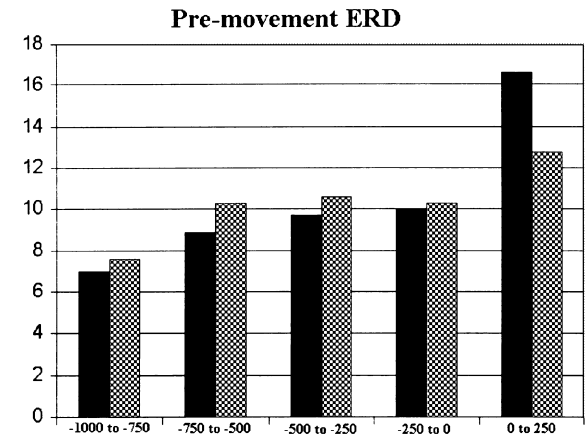

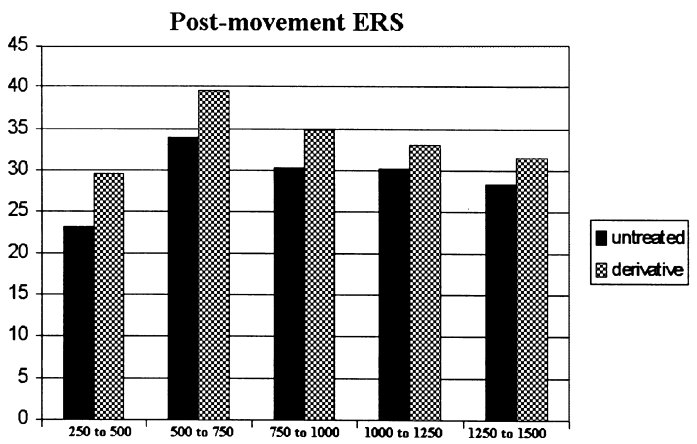

Fig. 3. (a) Dissimilarity between successive ERD time intervals, for the untreated MEG and for the MEG derivatives, both for the pre-movement ERD (lefthand diagram) and for the post-movement ERS (right-hand diagram). The dissimilarity, which is defined as 1 - absolute (correlation (map 1, map 2)), is larger for the ERD on untreated MEG in all comparisons. This confirms that the ERD on MEG derivatives has a higher spatial stability, as a visual inspection of Fig. 2 suggested. (b) Global Field Power for all ERD time intervals for the untreated MEG and for the MEG derivatives, both for the pre-movement ERD (left-hand diagram) and for the post-movement ERS (right-hand diagram). The Global Field Power is larger in the ERD on MEG derivatives in all time intervals but one, which supports the notion that the ERD on MEG derivatives is more focal than the ERD on untreated MEG.

Medical Informatics from the Technical University of Graz, Austria. The authors wish to thank Professor G. Pfurtscheller and Dr G. Edlinger for their help with the various stages of data processing. The authors further wish to thank Dr G. van Boxtel for his valuable comments on an earlier version of this paper.

\section{References}

Babiloni F, Carducci F, Del Gratta C, Roberti GM, Cincotti F, Bagni O, Romani GL, Rossini PM, Babiloni C. Multimodal Integration of High Resolution EEG, MEG and Functional Magnetic Resonance Data. Int J Bioelectromag 1999;1(1):9.

Edlinger G, Bastiaansen M, Brunia C, Neuper C, Pfurtscheller G. Cortical oscillatory activity assessed by combined EEG and MEG recordings and high-resolution ERD methods. Biomedizinische Technik 1999;44(2):131-134.

Hämäläinen MS, Ilmoniemi RJ. Interpreting magnetic fields of the brain: minimum norm estimates. Med Biol Eng Comput 1994;32:35-42.

Hari R, Salmelin R. Human cortical oscillations: a neuromagnetic view through the skull. Trends Neurosci 1997;20(1):44-49.

Lehtelä L, Salmelin R, Hari R. Evidence for reactive magnetic $10-\mathrm{Hz}$ rhythm in the human auditory cortex. Neurosci Lett 1997;222:111-114.
Lehmann D. Multichannel topography of human alpha EEG fields. Electroenceph clin Neurophysiol 1971;31:439-449.

Pfurtscheller G, Aranibar A. Event-related cortical desynchronization detected by power measurements of scalp EEG. Electroenceph clin Neurophysiol 1977;42:817-826.

Pfurtscheller G, Berghold A. Patterns of cortical activation during planning of voluntary movement. Electroenceph clin Neurophysiol 1989;72:250-258.

Pfurtscheller G, Lopez da Silva FH. Event Related Desynchronization, , Handbook of Electroencephalography and Clinical Neurophysiology (revised series), Vol. 6. Amsterdam: Elsevier, 1999.

Salmelin R, Hari R. Spatio-temporal characteristics of sensori-motor neuromagnetic rhythms related to thumb movement. Neuroscience 1994;60(2):537-550.

Salmelin R, Forss N, Knuutila J, Hari R. Bilateral activation of human somatomotor cortex by distal hand movements. Electroenceph clin Neurophysiol 1995a;95:444-452.

Salmelin R, Hämäläinen M, Kajola M, Hari R. Functional segregation of movement-related rhythmic activity in the human brain. Neuroimage 1995b;2:237-243.

Skrandies W. Visual information processing: topography of brain electrical activity. Biol Psychol 1995;40:1-15.

Tiihonen J, Hari R, Kajola M, Karhu J, Ahlfors S, Tissari S. Magnetoencephalographic $10 \mathrm{~Hz}$ rhythm from the human auditory cortex. Neurosci Lett 1995;129:303-305. 